\title{
Disease Spread over Randomly Switched Large-Scale Networks
}

\author{
Masaki Ogura $^{1}$ and Victor M. Preciado ${ }^{2}$
}

\begin{abstract}
In this paper we study disease spread over a randomly switched network, which is modeled by a stochastic switched differential equation based on the so called $\mathrm{N}$-intertwined model for disease spread over static networks. Assuming that all the edges of the network are independently switched, we present sufficient conditions for the convergence of infection probability to zero. Though the stability theory for switched linear systems can naively derive a necessary and sufficient condition for the convergence, the condition cannot be used for large-scale networks because, for a network with $n$ agents, it requires computing the maximum real eigenvalue of a matrix of size exponential in $n$. On the other hand, our conditions that are based also on the spectral theory of random matrices can be checked by computing the maximum real eigenvalue of a matrix of size exactly $n$.
\end{abstract}

\section{INTRODUCTION}

Network epidemiology, a branch of mathematical epidemiology which aims to mathematically understand disease spread over networks, has been attracting ever-growing attention [1]. One of the reasons comes from the finding that the heterogeneous structure of real world networks, such as an uneven distribution of the number of neighbors of individuals, cannot be ignored to perform an accurate prediction of epidemics [2]. Another reason comes from recent advancements of the technologies for collecting a massive amount of data from real human interactions, which enable researchers to build accurate mathematical models of human networks (see [3] and references therein). One of the recent achievements of network epidemiology, for example, is the effective prediction of the pandemic of H1N1 influenza on 2009 [4].

In the last decade, the major emphasis of network epidemiology has been on the disease spread over static networks [1]. This time-invariance assumption enables us to model disease spread as a time-homogeneous Markov process, for which an effective approximation by a constant-coefficient differential equation, called an $N$-intertwined model, is available [2], [5]. For example it is known [2], [5] that the evolution of a certain epidemic model over a static network is characterized by only the maximum real part of the eigenvalues of the adjacency matrix of the network.

However, recently, several simulation studies have reported that the dynamical nature of human interactions can greatly affect the way epidemics evolve and that static approximations of such dynamical networks may result in

\footnotetext{
${ }^{1} \mathrm{M}$. Ogura is with the Department of Mathematics and Statistics, Texas Tech University, TX 79409, USA. msk.ogura@gmail.com, masaki.ogura@ttu.edu

${ }^{2}$ V.M. Preciado is with the Department of Electrical and Systems Engineering at the University of Pennsylvania, Philadelphia, PA 19104, USA. preciado@seas. upenn. edu
}

inaccurate conclusions [6]. For example, Vazquez et al. [7] observe that the speed of disease spread can be substantially slowed down on randomly switched networks than on static ones. The result by Volz and Meyers [8] suggests that the frequency of the change of the topology of network structure is an important factor for the emergence of endemic. In [9], it is numerically confirmed that so-called memory effect of networks can slow down disease spread. Despite those simulation results, rigorous understanding of disease spread over dynamical networks based on mathematical analysis is very limited.

This paper gives an analysis of disease spread over randomly switched networks with the stability theory for switched linear systems called Markov jump linear systems [10] and the spectral theory of random graphs (see, e.g., [11]). We provide sufficient conditions for epidemics to eventually die out over randomly switched networks having independent and Markovian edges. This paper can be considered as a stochastic counter-part of the recently submitted paper [12], where the authors study epidemics over deterministically switched networks.

One of the major difficulties of the problem is in the computational cost. The stability theory for Markov jump linear systems in fact gives a necessary and sufficient condition for epidemics eventually dying out as we will see later (Proposition 3.1). Unfortunately, checking the condition requires finding the maximum real eigenvalue of a matrix with size $2^{n(n-1) / 2}$, where $n$ denotes the number of the agents in a network. On the other hand, the size of the matrix appearing in the proposed conditions is equal to $n$, which allows us to apply the conditions for large-scale networks.

This paper is organized as follows. After giving the notation used in this paper, in Section II we give the model of disease spread over randomly switched networks. Then Section III gives sufficient conditions for epidemics dying out over randomly switched networks having independent edges. Examples are presented in Section IV. Finally in Section V we extend the obtained results to the case when networks are modeled by weighted graphs.

\section{A. Mathematical Preliminaries}

We let $I_{n}$ denote the $n \times n$ identity matrix. The subscript $n$ will be omitted when it is obvious from the context. A real vector $v$ is said to be nonnegative, written $v \geq 0$, if it has only nonnegative entries. By $\mathbb{1}_{n}$ and $\mathbb{1}_{n, m}$ we denote the $n$-vector and the $n \times m$ matrix whose entries are all one. A square matrix is said to be Metzler if its off-diagonal entries are nonnegative. We denote the Kronecker product of matrices $A$ and $B$ by $A \otimes B$. 
The Euclidean norm of $x \in \mathbb{R}^{n}$ is denoted by $\|x\|$. Also we define the 1-norm of $x$ by $\|x\|_{1}=\sum_{i=1}^{n}\left|x_{i}\right|$. The maximum real part of the eigenvalues of $A$ is denoted by $\eta(A)$. We say that $A$ is Hurwitz stable if $\eta(A)<0$. The matrix measure [13] of $A \in \mathbb{R}^{n \times n}$ is defined by $\mu(A)=\lim _{h \rightarrow 0}(\|I+A h\|-1) / h$, where $\|\cdot\|$ denotes the maximum singular value. When $A$ is symmetric and thus has only real eigenvalues, its maximum eigenvalue is denoted by $\lambda_{\max }(A)$.

An undirected graph is a pair $\mathscr{G}=(\mathscr{V}, \mathscr{E})$, where $\mathscr{V}$ is a finite and nonempty set and $\mathscr{E}$ is a set consisting of distinct and unordered pairs $(i, j)$ of $i, j \in \mathscr{V}$. We call the elements of $\mathscr{V}$ vertexes and those of $\mathscr{E}$ edges. The adjacency matrix $A_{\mathscr{G}}$ of $\mathscr{G}$ is defined as the square $\{0,1\}$-matrix of size $|\mathscr{V}|$ whose $(i, j)$ entry is one if and only if $(i, j) \in \mathscr{E}$. Clearly the adjacency matrix of an undirected graph is symmetric and has zero diagonals. We say that $j \in \mathscr{V}$ is a neighbor of $i \in \mathscr{V}$ (or $i$ and $j$ are adjacent) if $(i, j) \in \mathscr{V}$. The degree of $i$ is defined as the number of the neighbors of $i$.

The expectation of a random variable is denoted by $E[\cdot]$. All the Markov processes appearing in this paper are assumed to have finite state spaces.

\section{EPIDEMIC MOdEL OVER RANDOMLY SWITCHED NETWORKS}

In this section we give a model for the spread of disease over randomly switched networks using switched differential equations. The model is based on a model called $N$ intertwined model originally proposed for epidemics over static networks [5]. Then we will review some results on the stability of switched linear systems that will be used in this paper.

\section{A. N-intertwined Model for Static Networks}

We first give an overview of the $N$-intertwined model [5] for so-called susceptible-infected-susceptible types of disease. Let a population be modeled by an undirected graph $\mathscr{G}$ over vertexes $\{1, \ldots, n\}$. We regard each vertex as an agent who can be infected and also can transmit disease to its neighbors. In the model, at each time $t \geq 0$, each vertex can be in one of the two states: susceptible or infected. We assume that, when a vertex $i$ is infected, the transition to the susceptible state occurs following a Poisson process with rate $\delta_{i}$, called curing rate. On the other hand, if $i$ is in the susceptible state and one if its neighbors is in the infected state, then $i$ will make a transition to the infected state by following a Poisson process with rate $\beta_{j}$, called infection rate. All the Poisson processes are assumed to be independent. Throughout this paper we assume that population has homogeneous infection rate $\beta$ and curing rate $\delta$, i.e., we assume that $\beta_{1}=\cdots=\beta_{n}=\beta$ and $\delta_{1}=\cdots=\delta_{n}=\delta$.

Let $p_{i}(t)$ denote the probability that the vertex $i$ is infected. Define $p=\left[\begin{array}{lll}p_{1} & \cdots & p_{n}\end{array}\right]^{\top}$ and $P=\operatorname{diag}\left(p_{1}, \ldots, p_{n}\right)$. Then the $N$-intertwined model [5] reads

$$
\frac{d p}{d t}=\left(\beta A_{\mathscr{G}}-\delta I\right) p-\beta P A_{\mathscr{G}} p,
$$

where $p(0)=p_{0} \in[0,1]^{n}$. We notice that clearly $p(t) \geq 0$ for every $t \geq 0$.

\section{B. Switched N-intertwined Model}

Based on the $N$-intertwined model (1) we can readily state our model of disease spread over randomly switched networks. We assume that our randomly switched network is modeled as $\mathscr{G}_{\sigma}:=\left\{\mathscr{G}_{\sigma(t)}\right\}_{t \geq 0}$, where $\sigma=\{\sigma(t)\}_{t \geq 0}$ is a timehomogeneous Markov process taking its values in $\{1, \ldots, N\}$ and $\mathscr{G}_{1}, \ldots, \mathscr{G}_{N}$ are undirected graphs over vertexes $\{1, \ldots, n\}$. Examples of such random switched graphs include the activity driven networks [14], graphs with edge swapping [8], and temporal exponential random graphs [15]. Then we model the disease spread over the randomly switched network $\mathscr{G}_{\sigma}$ by the switched $N$-intertwined model

$$
\Sigma: \frac{d p}{d t}=\left(\beta A_{\mathscr{G}_{\sigma(t)}}-\delta I\right) p-\beta P A_{\mathscr{G}_{\sigma(t)}} p,
$$

where $p(0)=p_{0} \in[0,1]^{n}$ and $\sigma(0)=\sigma_{0} \in\{1, \ldots, N\}$ are arbitrary constants.

The principal aim of this paper is to give conditions under which the zero equilibrium of $\Sigma$ is stable, i.e., the infection probability $p$ converges or stays close to the origin. We introduce the following definitions.

\section{Definition 2.1: We say that $\Sigma$ is}

1) mean stable if there exist $C>0$ and $\varepsilon>0$ such that, for every $p_{0}$ and $\sigma_{0}$, it holds that $E[\|p(t)\|] \leq C e^{-\varepsilon t}\left\|p_{0}\right\|$;

2) almost surely stable if, for every $p_{0}$ and $\sigma_{0}$, it holds that $P\left(\lim _{t \rightarrow \infty}\|p(t)\|=0\right)=1$.

The following linearized model

$$
\bar{\Sigma}: \frac{d \bar{p}}{d t}=\left(\beta A_{\mathscr{G}_{\sigma(t)}}-\delta I\right) \bar{p}
$$

of $\Sigma$ plays as an important role as the one for static networks does in [16], [17], where the authors propose the optimal vaccination strategy for the disease spread over static networks. We let the initial conditions of $\bar{\Sigma}$ given by constants $\bar{p}(0)=\bar{p}_{0} \in[0,1]^{n}$ and $\sigma(0)=\bar{\sigma}_{0} \in\{1, \ldots, N\}$. The next lemma is easy to see but fundamental.

Lemma 2.2: If $p_{0}=\bar{p}_{0}$ and $\sigma_{0}=\bar{\sigma}_{0}$, then we have $\|p(t)\|_{1} \leq\|\bar{p}(t)\|_{1}$ for every $t \geq 0$ with probability one.

Proof: Let $e(t)=\bar{p}(t)-p(t)$. Then $e(0)=0$ and also $d e / d t=\beta P A_{\mathscr{G}_{\sigma(t)}} p \geq 0$ because all of $\beta, P, A_{\mathscr{G}_{\sigma(t)}}$, and $p$ are nonnegative. Therefore $p(t) \leq \bar{p}(t)$ entry-wise for every $t \geq$ 0 . Finally multiply $\mathbb{1}_{n}^{\top}$ to the obtained inequality from the left to obtain the desired inequality.

Therefore, the study of the stability of $\Sigma$ can be reduced to that of the switched linear system $\bar{\Sigma}$, for which various results from the theory of switched linear systems [10] are available. We will review some of the results in the next section.

\section{Stability of Markov Jump Linear Systems}

As in the previous section let $\sigma=\{\sigma(t)\}_{t \geq 0}$ be a timehomogeneous Markov process taking its values in $\{1, \ldots, N\}$ 
and let $A_{1}, \ldots, A_{N} \in \mathbb{R}^{n \times n}$. Consider the switched linear system (called Markov jump linear system [10])

$$
\frac{d x}{d t}=A_{\sigma(t)} x
$$

where $x(0)=x_{0} \in \mathbb{R}^{n}$ and $\sigma(0)=\sigma_{0}$ are arbitrary constants. The stability of Markov jump linear systems is defined in the following standard way.

Definition 2.3: We say that (3) is

1) mean stable if there exist $C>0$ and $\varepsilon>0$ such that $E[\|x(t)\|] \leq C e^{-\varepsilon t}\left\|x_{0}\right\|$ for all $x_{0}$ and $\sigma_{0}$;

2) almost surely stable if, for all $x_{0}$ and $\sigma_{0}$, there holds $P\left(\lim _{t \rightarrow \infty}\|x(t)\|=0\right)=1$.

We say that the Markov jump linear system (3) is positive [18] if $x_{0} \geq 0$ implies $x(t) \geq 0$ for every $t \geq 0$ with probability one. For the system (3) to be positive, it is necessary and sufficient that all the matrices $A_{1}, \ldots, A_{N}$ are Metzler. For example, $\bar{\Sigma}$ defined in (2) is a positive Markov jump linear system. The next stability condition is obtained in [18].

Proposition 2.4: Assume that the Markov jump linear system (3) is positive. Let $\Pi \in \mathbb{R}^{N \times N}$ be the infinitesimal generator of $\sigma$. Then (3) is mean stable if and only if the matrix

$$
\mathscr{A}=\Pi^{\top} \otimes I_{n}+\operatorname{diag}\left(A_{1}, \ldots, A_{N}\right) \in \mathbb{R}^{n N \times n N}
$$

is Hurwitz stable.

We also recall the next proposition, which gives a sufficient condition for the almost sure stability of not necessarily positive Markov jump linear systems.

Proposition 2.5 ([19, Theorem 4.2]): Assume that $\sigma$ has the unique stationary distribution $\pi$. Then (3) is almost surely stable if $E\left[\mu\left(A_{\pi}\right)\right]<0$.

\section{Disease Spread OVER RAndomly Switched NETWORKS WITH INDEPENDENT EDGES}

The aim of this section is to give an easy to apply sufficient condition for the stability of the switched $N$-intertwined model $\Sigma$ under the assumption that the underlying randomly switched network has independent edges.

\section{A. Computational Difficulty}

Before presenting the main result we first observe the computational difficultly of the stability analysis. Theoretically, the next proposition completely solves the problem by giving a necessary and sufficient condition for mean stability via the eigenvalues of a matrix.

Proposition 3.1: Let $\Pi \in \mathbb{R}^{N \times N}$ be the infinitesimal generator of the Markov process $\sigma$. Define

$$
\mathscr{A}_{\beta}=\Pi^{\top} \otimes I_{n}+\beta \operatorname{diag}\left(A_{\mathscr{G}_{1}}, \ldots, A_{\mathscr{G}_{N}}\right) .
$$

Then, $\Sigma$ is mean stable if and only if $\eta\left(\mathscr{A}_{\beta}\right)<\delta$.

Proof: The Markov jump linear system $\bar{\Sigma}$ is positive because the matrix $\beta A_{\mathscr{G}_{i}}-\delta I$ is Metzler for every $i$. Therefore, using Proposition 2.4, one can see that $\eta\left(\mathscr{A}_{\beta}\right)<\delta$ implies that $\bar{\Sigma}$ is mean stable. Thus, by Lemma 2.2 , we can show that $\Sigma$ is also mean stable.

On the other hand, assume that $\Sigma$ is mean stable. Here we provide only a sketch of the proof. By Theorem 3.8 in [20], we can show that $\Sigma$ admits Lyapunov functions. Then it turns out that these Lyapunov functions also apply to $\bar{\Sigma}$ around a sufficiently small neighborhood of the origin. This is because, in such a neighborhood, the second order term $\beta P A_{G_{\sigma}} p$ of $\Sigma$ can be ignored compared with its linear term. Therefore we can conclude that $\bar{\Sigma}$ is also mean stable. Hence, again by Proposition 2.4, we obtain $\eta\left(\mathscr{A}_{\beta}\right)<\delta$.

Though Proposition 3.1 gives a practical characterization of stability when the size $n$ of a switched network is effectively small, unfortunately, it cannot be easily used for real world networks with a large $n$. The size of the matrix $\mathscr{A}_{\beta}$ equals $n N$, where $N$ is the number of all the possible configurations of the switched network. This $N$ can be very large when the network has many agents and can have various patterns of topology. In the extreme case when all the $\left(\begin{array}{l}n \\ 2\end{array}\right)=n(n-1) / 2$ possible edges can be present or not independently, $N$ equals the rapidly growing exponential $2^{n(n-1) / 2}$. By the same reason, Proposition 2.5 is often not easy to use in practice when $n$ is large because it involves finding the matrix measure of a potentially huge number of matrices. We also remark that, in such a situation, it would be computationally too expensive to even estimate a stability condition by simulation.

These difficulties motivate us to find easy to apply sufficient conditions for stability when the size of a network is large.

\section{B. Stability Condition for Switched Edge-independent Net- works}

One of the classical models of large-scale and random but static networks is Erdős-Rényi graphs [21], in which edges are assigned uniformly and independently for each pair of vertexes. Based on the fact that the distributions of the degrees of Erdős-Rényi graphs shows a large deviation from those of real world networks, recently Chung [11] proposed an improved version of the graphs by removing the uniformity constraint.

Extending the above models, in this paper we study the disease spread over edge-independent random dynamical networks defined as follows.

Definition 3.2: For distinct $i, j \in\{1, \ldots, n\}$ we let $A_{i j}=\left\{A_{i j}(t)\right\}_{t \geq 0}$ denote the $(i, j)$-element of the matrixvalued stochastic process $A_{\mathscr{G}_{\sigma}}$. We say that $\mathscr{G}_{\sigma}$ has independent edges if the processes $\left\{A_{i j}\right\}_{i>j}$ are stochastically independent.

Then our assumption on the randomly switched network $\mathscr{G}_{\sigma}$ can be stated as follows.

Assumption 3.3: $\mathscr{G}_{\sigma}$ has independent edges and $\sigma$ has a unique stationary distribution.

Remark 3.4: The existence of a unique stationary distribution is not very restrictive because a Markov process in general has a unique stationary distribution under a mild assumption of irreducibility and recurrence property. 
Let $\pi$ denote the stationary distribution of $\sigma$. Then $\mathscr{G}_{\pi}$ is the random stationary graph with the random adjacency matrix $A_{\mathscr{G}_{\pi}}$. Define $\bar{A}=E\left[A_{\mathscr{G}_{\pi}}\right]$ and

$$
\Delta=\max _{1 \leq i \leq n}\left(\sum_{j=1}^{n} \bar{A}_{i j}\left(1-\bar{A}_{i j}\right)\right)
$$

Without loss of generality we assume $\Delta>0$, because otherwise $\Delta=0$ and therefore the graph process $\mathscr{G}_{\sigma}$ equals the static network having the $\{0,1\}$-matrix $\bar{A}$ as its adjacency matrix. In this case $\Sigma$ coincides with the $N$-intertwined model (1) for static networks and therefore $\Sigma$ is stable if and only if $\lambda_{\max }(\bar{A})<\delta / \beta$ as found in [5].

The next theorem gives an easy-to-use alternative of Proposition 3.1 and is the main result of this paper.

Theorem 3.5: Define the function $f$ on $[0, \infty)$ by

$$
f(s)=s+2 n^{2} \exp \left(-\frac{3 s^{2}}{2 s+6 \Delta}\right) .
$$

Then $\Sigma$ is almost surely stable if

$$
\lambda_{\max }(\bar{A})+\min _{s \geq 0} f(s)<\delta / \beta .
$$

Remark 3.6: We can understand the quantity $\min _{s \geq 0} f(s)$ as the measure of uncertainty because the quantity increases with respect to $\Delta$, which measures the variance of the stationary random graph $\mathscr{G}_{\pi}$.

We note that the size of the matrix $\bar{A}$ in the condition (6) is only $n$ and is much smaller than that of $\mathscr{A}_{\beta}$ in Proposition 3.1. Also $\bar{A}$ can be found as follows. Since $\mathscr{G}_{\sigma}$ has independent edges, each scalar-valued stochastic process $A_{i j}$ is a $\{0,1\}$-valued time-homogeneous Markov process. Then its infinitesimal generator is of the form

$$
\left[\begin{array}{cc}
-p_{i j} & p_{i j} \\
q_{i j} & -q_{i j}
\end{array}\right]
$$

for some $p_{i j}, q_{i j} \geq 0$. The uniqueness of the stationary distribution of $\sigma$ yields that $p_{i j}+q_{i j}>0$. Then we can easily show that the stationary distribution of $A_{i j}$, denoted by $\pi_{i j}$, is given by $\pi_{i j}(\{0\})=q_{i j} /\left(p_{i j}+q_{i j}\right)$ and $\pi_{i j}(\{1\})=p_{i j} /\left(p_{i j}+q_{i j}\right)$. Therefore we obtain $\bar{A}_{i j}=p_{i j} /\left(p_{i j}+q_{i j}\right)$ when $i \neq j$ and $\bar{A}_{i i}=0$ for every $i$.

Moreover, the next proposition shows that the minimum $\min _{s \geq 0} f(s)$ in (6) can be found by solving a convex program.

Proposition 3.7: There exists $0<s_{0}<2 \Delta$ such that $f$ is convex on $\left[s_{0}, \infty\right)$ and

$$
\min _{s \geq 0} f(s)=\min \left(f(0), \min _{s \geq s_{0}} f(s)\right) \text {. }
$$

\section{Proofs}

In this section we give the proofs of Theorem 3.5 and Proposition 3.7. For the proof of Theorem 3.5 we state two propositions.

Proposition 3.8: If $E\left[\lambda_{\max }\left(A \mathscr{G}_{\pi}\right)\right]<\delta / \beta$, then $\Sigma$ is almost surely stable.

Proof: Recall [13] that, if $A$ is a real symmetric matrix, then $\mu(A)=\lambda_{\max }(A)$. Thus we have $\mu\left(A_{\mathscr{G}_{\pi}}\right)=\lambda_{\max }\left(A_{\mathscr{G}_{\pi}}\right)$ with probability one. Now assume $E\left[\lambda_{\max }\left(A_{\mathscr{G}_{\pi}}\right)\right]<\delta / \beta$. Then $E\left[\mu\left(\beta A \mathscr{G}_{\pi}-\delta I\right)\right]=E\left[\lambda_{\max }\left(\beta A_{\mathscr{G}_{\pi}}-\delta I\right)\right]<0$. Therefore, by Proposition $2.5, \bar{\Sigma}$ is almost surely stable; i.e., $\|\bar{p}(t)\| \rightarrow 0$ as $t \rightarrow \infty$ with probability one. Therefore, by using the equivalence of the norms $\|\cdot\|$ and $\|\cdot\|_{1}$ and also Lemma 2.2, we can show the almost sure stability of $\Sigma$.

To evaluate $E\left[\lambda_{\max }\left(A \mathscr{G}_{\pi}\right)\right]$ we will need the following result from the spectral theory of random graphs.

Proposition 3.9 ([22, p. 7]): Let $\mathscr{G}$ be a random undirected graph on the vertex set $\{1, \ldots, n\}$, where two vertexes are adjacent in $\mathscr{G}$ independently. Let $\bar{A}=E\left[A_{\mathscr{G}}\right]$ and define $\Delta$ by (4). Then, for every $s \geq 0$,

$$
P\left(\left\{\lambda_{\max }(A)>\lambda_{\max }(\bar{A})+s\right\}\right) \leq 2 n \exp \left(-\frac{3 s^{2}}{2 s+6 \Delta}\right) .
$$

Now we prove Theorem 3.5.

Proof of Theorem 3.5: Notice that the function $f$ is well defined. Since $\mathscr{G}_{\sigma}$ has independent edges, any pair of vertexes are adjacent independently with others in the random graph $\mathscr{G}_{\pi}$. Therefore Proposition 3.9 applies to $\mathscr{G}_{\pi}$. Let $\Omega$ denote the underlying probability space and define, for each $s \geq 0$,

$$
\Omega_{s}=\left\{\omega \in \Omega: \lambda_{\max }\left(A \mathscr{G}_{\pi}\right)>\lambda_{\max }(\bar{A})+s\right\} .
$$

If $\omega \in \Omega_{s}^{c}$, then $\lambda_{\max }\left(A_{\mathscr{G}_{\pi}}\right) \leq \lambda_{\max }(\bar{A})+s$. On the other hand, if $\omega \in \Omega_{s}$, then we have the trivial estimate $\lambda_{\max }\left(A_{\mathscr{G}_{\pi}}\right)<n$. Therefore, by (8) we obtain

$$
\begin{aligned}
E\left[\lambda_{\max }\left(A_{\mathscr{G}_{\pi}}\right)\right] & <P\left(\Omega_{s}^{c}\right)\left(\lambda_{\max }(\bar{A})+s\right)+P\left(\Omega_{s}\right) n \\
& \leq \lambda_{\max }(\bar{A})+s+2 n^{2} \exp \left(-\frac{3 s^{2}}{2 s+6 \Delta}\right) \\
& =\lambda_{\max }(\bar{A})+f(s) .
\end{aligned}
$$

Since $f$ is continuous and also $f(s)$ diverges to $+\infty$ as $s \rightarrow \infty$, the minimum $\min _{s \geq 0} f(s)$ exists. Therefore, taking the minimum with respect to $s \geq 0$ on the most right hand side of (9) proves $E\left[\lambda_{\max }\left(A_{\mathscr{G}_{\pi}}\right)\right] \leq \lambda_{\max }(\bar{A})+\min _{s \geq 0} f(s)$. Hence, if (6) holds, then $\Sigma$ is almost surely stable by Proposition 3.8.

Then we give the proof of Proposition 3.7.

Proof of Proposition 3.7: Let us only prove the convexity part, as the equation (7) follows from a straightforward argument. Throughout this proof we shall work with the translation of $f$ defined by $g(s)=f(s-3 \Delta)(s \geq 3 \Delta)$. We need to show that there exists $3 \Delta<s_{0}<5 \Delta$ such that $g$ is convex on $\left[s_{0}, \infty\right)$. Define $h_{1}, h_{2}:[3 \Delta, \infty) \rightarrow \mathbb{R}$ by

$h_{1}(s)=\frac{c_{1}\left(c_{2} s^{2}-c_{3}+\sqrt{2 c_{3} s}\right)}{s^{4} \exp \left(c_{2} s+c_{3} s^{-1}\right)}, h_{2}(s)=c_{2} s^{2}-c_{3}-\sqrt{2 c_{3} s}$,

where $c_{1}=2 n^{2} e^{9 \Delta}, c_{2}=3 / 2$, and $c_{3}=27 \Delta^{2} / 2$. Then we can easily show that $g^{\prime \prime}=h_{1} h_{2}$. It is trivial to check that $h_{1}(s) \geq 0$. Also, since $h_{2}(3 \Delta)<0$ and $h_{2}^{\prime}(s)>0$, there exists $s_{0}>3 \Delta$ such that $h_{2}<0$ on $\left[3 \Delta, s_{0}\right)$ and $h_{2}>0$ on $\left(s_{0}, \infty\right)$. Since $h(5 \Delta)=24 \Delta^{2}-3 \sqrt{15} \Delta>(24-3 \sqrt{15}) \Delta^{2}>0$, we can check $s_{0}<5 \Delta$. The above argument yields $g^{\prime \prime}<0$ on $\left[3 \Delta, s_{0}\right)$, as desired. Then we can prove (7) by carefully investigating the derivatives $g^{\prime}$ and $g^{\prime \prime}$. The details are omitted. 


\section{EXAMPLES}

In this section we apply Theorem 3.5 to the following important classes of dynamical graphs: randomly switched graphs with communities [23] and expected degrees [11].

\section{A. Switched Graphs with Community Structure}

Let us consider a population grouped into the two communities $\mathscr{V}_{1}=\left\{1, \ldots, n_{1}\right\}$ and $\mathscr{V}_{2}=\left\{n_{1}+1, \ldots, n_{1}+n_{2}\right\}$. We assume that, for any pair of vertexes $(i, j)$, the edgeprocess $A_{i j}$ has the stationary distribution $\mu_{i j}$ that depends only on the communities to which $i$ and $j$ belong. Therefore, there exist $\theta_{1}, \theta_{2}, \phi \in[0,1]$ such that

$$
\mu_{i j}(\{1\})= \begin{cases}\theta_{\ell} & i, j \in \mathscr{V}_{\ell}, \ell=1,2 \\ \phi & \text { otherwise. }\end{cases}
$$

Then it follows that

$$
\bar{A}=\left[\begin{array}{cc}
\theta_{1} \mathbb{1}_{n_{1}, n_{1}}-\theta_{1} I_{n_{1}} & \phi \mathbb{1}_{n_{1}, n_{2}} \\
\phi \mathbb{1}_{n_{2}, n_{1}} & \theta_{2} \mathbb{1}_{n_{2}, n_{2}}-\theta_{2} I_{n_{2}}
\end{array}\right]
$$

Therefore

$$
\begin{array}{r}
\Delta=\max \left(\left(n_{1}-1\right) \theta_{1}\left(1-\theta_{1}\right)+n_{2} \phi(1-\phi),\right. \\
\left.\left(n_{2}-1\right) \theta_{2}\left(1-\theta_{2}\right)+n_{1} \phi(1-\phi)\right) .
\end{array}
$$

Also we can show that

$$
\lambda_{\max }(\bar{A})=\frac{n_{1} \theta_{1}+n_{2} \theta_{2}+\sqrt{\left(n_{1} \theta_{1}-n_{2} \theta_{2}\right)^{2}+4 n_{1} n_{2} \phi^{2}}}{2}-\varepsilon
$$

for some $\varepsilon$ lying in between $\theta_{1}$ and $\theta_{2}$.

For example let $n_{1}=10^{4}, n_{2}=10^{5}, \theta_{1}=0.5, \theta_{2}=0.3$, and $\phi=0.1$. Then one can compute $\lambda_{\max }(\bar{A})=3.04 \cdot 10^{4}$. Also, by solving the convex program (7), we can easily find $\max _{s>0} f(s)=9.83 \cdot 10^{2}$, which is effectively smaller than $\lambda_{\max }(\bar{A})$. Then, by Theorem $3.5, \Sigma$ is almost surely stable if $3.14 \cdot 10^{4}<\delta / \beta$. We remark that, in this case, it is almost impossible to use Proposition 3.1 because the matrix $\mathscr{A}_{\beta}$ has the dimension more than $10^{10^{9}}$.

\section{B. Switched Graphs with Expected Degrees}

In this section we study the special case when the expected adjacency matrix $\bar{A}$ has the following structure.

Assumption 4.1: There exist $\alpha_{1}, \ldots, \alpha_{n} \geq 0$ such that

$$
\bar{A}_{i j}=\alpha_{i} \alpha_{j}
$$

for every distinct pair $(i, j)$.

Epidemiologically, one can regard the constant $\alpha_{i}$ as the measure of the activity of the vertex $i$. Then we can understand Assumption 4.1 as stating that the frequency of the interaction between two agents $i$ and $j$ is solely determined by those activity measure.

Also Assumption 4.1 can be supported by its connection to one of the well known models of random graphs. Let $d \in \mathbb{R}^{n}$ be nonzero and nonnegative and let $\rho=1 /\left(\sum_{i=1}^{n} d_{i}\right)$. We say that an undirected random graph $\mathscr{G}$ has expected degrees $d$ [11] if edges are independently assigned to each pair of vertexes $(i, j)$ with probability $\rho d_{i} d_{j}$. We can show the next proposition under Assumptions 3.3 and 4.1. The proof is straightforward and is hence omitted.
Proposition 4.2: For each $i$ let $d_{i}=\alpha_{i} \sum_{j=1}^{n} \alpha_{j}$. The stationary graph $\mathscr{G}_{\pi}$ has expected degrees $d$.

The next theorem gives a sufficient condition for the almost sure stability of $\Sigma$ in terms of the expected degrees of the stationary graph $\mathscr{G}_{\pi}$.

Theorem 4.3: Let

$$
\tilde{d}=\rho \sum_{i=1}^{n} d_{i}^{2}, \Delta_{d}=\max _{1 \leq i \leq n} \sum_{j=1}^{n} \rho d_{i} d_{j}\left(\rho-d_{i} d_{j}\right)
$$

and define the function $f$ by (5) with $\Delta$ replaced by $\Delta_{d}$. Then $\Sigma$ is almost surely stable if

$$
\tilde{d}+\min _{s \geq 0} f(s) \leq \delta / \beta
$$

Moreover $\min _{s \geq 0} f(s)$ satisfies (7).

Proof: It is straightforward to see that, for the random graph $\mathscr{G}_{\pi}$, the quantity $\Delta$ defined in (4) equals $\Delta_{d}$. Moreover, since $\bar{A}=\rho d d^{\top}$, we can show $\lambda_{\max }(\bar{A})=\tilde{d}$. Thus the sufficient condition (10) immediately follows from (6). The proof of the latter claim is exactly the same as that of Proposition 3.7.

Example 4.4: One of the well-used model of the degree sequences is those with power law distributions [11], [22]. We say that a degree sequence $d \in \mathbb{R}^{n}$ has the power law distribution with the power law exponent $\beta>2$, maximum degree $\Delta>0$, and average degree $\bar{d}>0$ if

$$
d_{i}=c\left(i+i_{0}-1\right)^{-1 /(\beta-1)}
$$

for every $i$, where

$$
c=\frac{\beta-2}{\beta-1} \bar{d} n^{1 /(\beta-1)}, i_{0}=n\left(\frac{\bar{d}(\beta-2)}{m(\beta-1)}\right)^{\beta-1} .
$$

For example let $n=10^{7}, \beta=2.2, \Delta=5 \cdot 10^{5}$, and $\bar{d}=10^{3}$. Then we have $\tilde{d}=3.15 \cdot 10^{4}$. Also, by solving the convex program (7) we obtain $\min _{s \geq 0} f(s)=1.97 \cdot 10^{3}$. Therefore, by Theorem 4.3, $\Sigma$ is almost surely stable if $3.35 \cdot 10^{4}<\delta / \beta$. We remark that the quantity $\min _{s \geq 0} f(s)$ measuring uncertainty is relatively small compared with $\lambda_{\max }(\bar{A})$.

\section{Disease SPREAd OVER RANDOMLy SWITCHED NETWORKS WITH WEIGHTS}

In this section, we extend the result obtained in Section III to the networks modeled by weighted graphs. We start by giving necessary definitions.

Definition 5.1: Let $\mathscr{V}$ be a finite set and let $\mathscr{E}$ be the set of all the distinct and unordered pairs of the elements of $\mathscr{V}$. Also let $w: \mathscr{E} \rightarrow[0, \infty):(i, j) \mapsto w(i, j)$ be a function. We call the pair $(\mathscr{V}, w)$ a weighted undirected graph. An element of $\mathscr{V}(\mathscr{E})$ is called a vertex (edge, respectively). For an edge $e \in \mathscr{E}$, We call $w(e)$ the weight of $e$.

Let $\mathscr{V}=\{1, \ldots, n\}$. The adjacency matrix $A_{(\mathscr{V}, w)} \in \mathbb{R}^{n \times n}$ of an weighted undirected graph $(\mathscr{V}, w)$ is defined by $\left[A_{(\mathscr{V}, w)}\right]_{i j}=w(i, j)$ for $i \neq j$ and $\left[A_{(\mathscr{V}, w)}\right]_{i i}=0$ for every $i$. When no confusion arises we write $A_{(\mathscr{V}, w)}$ as $A_{w}$. We notice that $A_{w}$ is symmetric because $(i, j)$ and $(j, i)$ are the same unordered pairs. Then we call the differential equation

$$
\frac{d p}{d t}=\left(\beta A_{w}-\delta I\right) p-\beta P A_{w} p
$$


the $N$-intertwined model of disease spread over $(\mathscr{V}, w)$. Epidemiologically, the weight $w(i, j)$ expresses the strength of the communication between the agents $i$ and $j$, in the sense that instantaneous rate that disease transmits from $i$ to $j$ (provided $i$ is infected and $j$ is not) equals the product $\beta w(i, j)$. This in particular implies that, without loss of generality, we can normalize $w$ as

$$
w(i, j) \leq 1
$$

by taking $\beta$ sufficiently large.

Then, as in Section II-B, let us assume that the weight of the given network of agents changes over time according to a time-homogeneous Markov process, i.e., suppose that there exists a time-homogeneous Markov process $\sigma=\{\sigma(t)\}_{t \geq 0}$ such that the weight of the graph at time $t \geq 0$ is given by the function $w_{\sigma(t)}: \mathscr{E} \rightarrow[0, \infty):(i, j) \mapsto w_{\sigma(t)}(i, j)$. Then we can model the disease spread over time-varying, weighted, and undirected graph $\mathscr{G}_{\sigma}:=\left(\mathscr{V}, w_{\sigma}\right)$ by the switched differential equation

$$
\Sigma_{w}: \frac{d p}{d t}=\left(\beta A_{w_{\sigma(t)}}-\delta I\right) p-\beta P A_{w_{\sigma(t)}} p .
$$

The almost sure stability of $\Sigma_{w}$ is defined in the same way as Definition 2.1. Also, extending Definition 3.2, We say that $\mathscr{G}_{\sigma}$ has independent edges if the stochastic processes $\left\{w_{\sigma(\cdot)}(i, j)\right\}_{(i, j) \in \mathscr{E}}$ are independent.

If $\sigma$ has a stationary distribution $\pi$, then we let $\operatorname{Var}\left(A_{w_{\pi}}\right)$ be the $n \times n$ real matrix obtained by taking the variances of the random matrix $A_{w_{\pi}}$ entry-wise. Since $\sigma$ is assumed to have finitely many states, each entry of $A_{w_{\pi}}$ is a distribution having finite support so that it is straightforward to find $\operatorname{Var}\left(A_{w_{\pi}}\right)$. The next theorem extends Theorem 3.5 to the weighted and randomly switched networks.

Theorem 5.2: Assume that $\mathscr{G}_{\sigma}$ has independent edges and $\sigma$ has the unique stationary distribution $\pi$. Let $\bar{A}=E\left[A_{w_{\pi}}\right]$ and also let $\Delta$ be the maximum row sum of $\operatorname{Var}\left(A_{w_{\pi}}\right)$. Define the function $f$ by (5). If (6) holds, then $\Sigma_{w}$ is almost surely stable. Moreover the minimum $\min _{s \geq 0} f(s)$ can be found by solving the convex program (7).

Proof: From [22] we can easily check that Proposition 3.9 holds for a weighted random graph as long as its weights are all less than one with probability one and we replace $\Delta$ by the maximum row sum of $\operatorname{Var}\left(A_{\mathscr{G}}\right)$. Therefore, under the normalization (11), we can apply Proposition 3.9 to the stationary random graph $\mathscr{G}_{\pi}$ and prove the theorem in the same way as Theorem 3.5. The details of the proof are omitted.

\section{CONCLUSION}

We studied the disease spread over randomly switched networks having stochastically independent edges. The disease spread was modeled by a switched version of the $N$-intertwined model for static networks. Using the stability theory of Markov jump linear systems and the spectral theory of random matrices, we gave sufficient conditions for epidemics dying out almost surely. We can check the proposed conditions by finding the maximum real eigenvalue of a matrix whose size equal the number of the agents and thus is highly efficient compared with another condition based solely on the stability theory. We also gave an extension to the case when networks are modeled by weighted graphs.

\section{REFERENCES}

[1] R. Pastor-Satorras, C. Castellano, P. Van Mieghem, and A. Vespignani, "Epidemic processes in complex networks," 2014. [Online]. Available: http://arxiv.org/abs/1408.2701

[2] D. Chakrabarti, Y. Wang, C. Wang, J. Leskovec, and C. Faloutsos, "Epidemic thresholds in real networks," ACM Transactions on Information and System Security, vol. 10:13, 2008.

[3] A. Vespignani, "Predicting the behavior of techno-social systems." Science, vol. 325, pp. 425-428, 2009.

[4] D. Balcan, H. Hu, B. Goncalves, P. Bajardi, C. Poletto, J. J. Ramasco, D. Paolotti, N. Perra, M. Tizzoni, W. Van den Broeck, V. Colizza, and A. Vespignani, "Seasonal transmission potential and activity peaks of the new influenza $\mathrm{A}(\mathrm{H} 1 \mathrm{~N} 1)$ : a Monte Carlo likelihood analysis based on human mobility." BMC medicine, vol. 7:45, 2009.

[5] P. Van Mieghem, J. Omic, and R. Kooij, "Virus spread in networks," IEEE/ACM Transactions on Networking, vol. 17, pp. 1-14, 2009.

[6] N. Masuda and P. Holme, "Predicting and controlling infectious disease epidemics using temporal networks." F1000prime reports, vol. 5:6, 2013

[7] A. Vazquez, B. Rácz, A. Lukács, and A.-L. Barabási, "Impact of nonPoissonian activity patterns on spreading processes," Physical Review Letters, vol. 98, 158702, 2007.

[8] E. Volz and L. A. Meyers, "Epidemic thresholds in dynamic contact networks." Journal of the Royal Society, Interface / the Royal Society, vol. 6, pp. 233-241, 2009.

[9] K. Sun, A. Baronchelli, and N. Perra, "Epidemic spreading in non-Markovian time-varying networks," 2014. [Online]. Available: http://arxiv.org/abs/1404.1006

[10] O. L. Costa, M. D. Fragoso, and M. G. Todorov, Continuous-time Markov Jump Linear Systems. Springer, 2013.

[11] F. Chung, L. Lu, and V. Vu, "Spectra of random graphs with given expected degrees," Proceedings of the National Academy of Sciences of the United States of America, vol. 100, pp. 6313-6318, 2003.

[12] M. A. Rami, V. S. Bokharaie, O. Mason, and F. R. Wirth, "Stability criteria for SIS epidemiological models under switching policies," 2014. [Online]. Available: http://arxiv.org/abs/1306.0135

[13] C. Desoer and H. Haneda, "The measure of a matrix as a tool to analyze computer algorithms for circuit analysis," IEEE Transactions on Circuit Theory, vol. 19, pp. 480-486, 1972.

[14] N. Perra, B. Gonçalves, R. Pastor-Satorras, and A. Vespignani, "Activity driven modeling of time varying networks." Scientific reports, vol. 2:469, 2012.

[15] S. Hanneke, W. Fu, and E. P. Xing, "Discrete temporal models of social networks," Electronic Journal of Statistics, vol. 4, pp. 585-605, 2010.

[16] V. M. Preciado, M. Zargham, C. Enyioha, A. Jadbabaie, and G. Pappas, "Optimal vaccine allocation to control epidemic outbreaks in arbitrary networks," in 52nd IEEE Conference on Decision and Control. IEEE, 2013, pp. 7486-7491.

[17] V. M. Preciado, M. Zargham, C. Enyioha, A. Jadbabaie, and G. J. Pappas, "Optimal resource allocation for network protection against spreading processes," IEEE Transactions on Control of Network Systems, vol. 1, pp. 99-108, 2014

[18] M. Ogura and C. F. Martin, "Stability analysis of positive semiMarkovian jump linear systems with state resets," SIAM Journal on Control and Optimization, vol. 52, pp. 1809-1831, 2014.

[19] Y. Fang and K. Loparo, "Stabilization of continuous-time jump linear systems," IEEE Transactions on Automatic Control, vol. 47, pp. 1590$1603,2002$.

[20] R. Khasminskii, C. Zhu, and G. Yin, "Stability of regime-switching diffusions," Stochastic Processes and their Applications, vol. 117, pp. 1037-1051, 2007.

[21] L. Erdős and A. Rényi, "On random graphs. I," Publicationes Mathematicae, vol. 6, pp. 290-297, 1959.

[22] F. Chung and M. Radcliffe, "On the spectra of general random graphs," The Electronic Journal of Combinatorics, vol. 18, \#P215, 2011.

[23] S. Fortunato, "Community detection in graphs," Physics Reports, vol. 486, pp. $75-174,2010$ 